\title{
Dynamo-free plasma in the reversed-field pinch: Advances in understanding the reversed-field pinch improved confinement mode ${ }^{a)}$
}

\author{
J. K. Anderson, ${ }^{1, b)}$ J. Adney, ${ }^{1}$ A. Almagri, ${ }^{1}$ A. Blair, ${ }^{1} D_{\text {. }}$ L. Brower ${ }^{2}$ M. Cengher,${ }^{1}$ \\ B. E. Chapman, ${ }^{1}$ S. Choi, ${ }^{1}$ D. Craig, ${ }^{1}$ D. R. Demers, ${ }^{4}$ D. J. Den Hartog, ${ }^{1}$ B. Deng, ${ }^{2}$ \\ W. X. Ding, ${ }^{2}$ F. Ebrahimi, ${ }^{1}$ D. Ennis, ${ }^{1}$ G. Fiksel, ${ }^{1}$ C. B. Forest, ${ }^{1}$ P. Franz,${ }^{5}$ J. Goetz, ${ }^{1}$ \\ R. W. Harvey ${ }^{3}$ D. Holly, ${ }^{1}$ B. Hudson, ${ }^{1}$ M. Kaufman, ${ }^{1}$ T. Lovell, ${ }^{1}{ }^{1}$ L. Marrelli, ${ }^{5}$ P. Martin, ${ }^{5}$ \\ K. McCollam, ${ }_{1}^{1}$ V. V. Mirnov ${ }_{5}^{1}$ P. Nonn,${ }^{1}$ R. O'Connell, ${ }^{1}$ S. Oliva,${ }^{1}$ P. Piovesan, ${ }^{5}$ \\ S. C. Prager, ${ }^{1}$ I. Predebon, ${ }^{5}$ J. S. Sarff, ${ }^{1}$ G. Spizzo, ${ }^{5}$ V. Svidzinski, ${ }^{1}$ M. Thomas, ${ }^{1}$ and \\ M. D. Wyman ${ }^{1}$ \\ ${ }^{1}$ The University of Wisconsin, Madison, Wisconsin and the Center for Magnetic Self-Organization in \\ Laboratory and Astrophysical Plasmas \\ ${ }^{2}$ The University of California at Los Angeles, Los Angeles, California 90095 \\ ${ }^{3}$ CompX, Del Mar, California 92014 \\ ${ }^{4}$ Rensselaer Polytechnic Institute, Troy, New York 12180 \\ ${ }^{5}$ Consorzio RFX, Associazione EURATOM-ENEA sulla Fusione, Padova, Italy
}

(Received 19 November 2004; accepted 4 February 2005; published online 28 April 2005)

\begin{abstract}
Generation and sustainment of the reversed field pinch (RFP) magnetic configuration normally relies on dynamo activity. The externally applied electric field tends to drive the equilibrium away from the relaxed, minimum energy state which is roughly described by a flat normalized parallel current density profile and is at marginal stability to tearing modes. Correlated fluctuations of magnetic field and velocity create a dynamo electric field which broadens the parallel current density profile, supplying the necessary edge current drive. These pervasive magnetic fluctuations are also responsible for destruction of flux surfaces, relegating the standard RFP to a stochastic-magnetic transport-limited device. Application of a tailored electric field profile (which matches the relaxed current density profile) allows sustainment of the RFP configuration without dynamo-driven edge current. The method used to ascertain that a dynamo-free RFP plasma has been created is reported here in detail. Several confinement improvements during the accompanying periods of low magnetic fluctuations are observed. Namely, the magnetic fluctuation level is reduced to the point where stochastic-magnetic transport is no longer the dominant process in the core and nested flux surfaces are restored in the core of the dynamo-free RFP. (c) 2005 American Institute of Physics. [DOI: 10.1063/1.1883666]
\end{abstract}

\section{INTRODUCTION}

The reversed field pinch is a simple toroidal plasma created by applying a toroidal electric field within a relatively weak toroidal magnetic field and close fitting conducting shell. ${ }^{1,2}$ The resulting magnetic equilibrium (where the toroidal magnetic field decreases monotonically with minor radius and reverses direction in the plasma edge) relies on magnetic relaxation for sustainment. This is predicted by Taylor, ${ }^{3}$ where a minimum energy state (with the constraint of conservation of global helicity) has a flat normalized parallel current density $\left(J_{\|} / B=\right.$ const). This requires substantial poloidal current drive in the edge which is not provided by the applied electric field. ${ }^{4}$ The dynamo effect-the selfgeneration of current-is responsible for the necessary parallel current drive in the edge. ${ }^{5}$

The reversed field pinch (RFP) equilibrium has closely spaced rational surfaces where resonant tearing instabilities create magnetic perturbations. The toroidal induction tends to create a very peaked parallel current density profile whose

\footnotetext{
a) Paper EI1 6, Bull. Am. Phys. Soc. 49, 99 (2004).

${ }^{b)}$ Invited speaker.
}

gradient supplies energy to the tearing modes. Nonlinear interaction of the modes produces dynamo and flattens the current profile by driving parallel current in the edge and suppressing it in the core. The experimental relaxed state (modified from Taylor's prediction as the boundary current density must vanish) is when the dynamo has sufficiently flattened the $J_{\|} / B$ profile to marginally stabilize the dominant tearing modes. When the dynamo current drive is strong (as is the case in the standard RFP), the magnetic fluctuations are large enough so that islands on adjacent resonant surfaces overlap and create large radial bands of magnetic stochasticity. The symmetry of nested magnetic surfaces is destroyed and the confinement is dramatically lower than classical predictions.

With application of an inductive electric field profile that is tailored to match the current density profile of the relaxed state, the parallel edge current normally supplied by the dynamo is no longer needed to sustain the RFP configuration. These plasmas are called "dynamo free," as the applied electric field profile does account for the current density over nearly the entire cross section. The applied electric field is intended to flatten the $J_{\|} / B$ profile by driving parallel current 
in the edge, thereby providing stabilization to tearing modes. ${ }^{6}$ Both the $J_{\|}$and $\mathbf{B}$ profiles are modified ${ }^{7,8}$ (including a substantial central peaking of the parallel current density), $J_{\|} / B$ undergoes a relatively subtle change but does stabilize the plasma to tearing modes, and the mode amplitudes are observed to decrease dramatically. This narrows the islands to the point where they no longer overlap, and there is an increase in confinement consistent with the restoration of nested flux surfaces in the plasma core.

This paper consists of two parts. First, a demonstration that the reversed field pinch can exist without a dynamo is presented. A study of the parallel Ohm's law is used to quantify the level of dynamo activity: a plasma with zero dynamo activity is described by a simple form $E_{\|}=\eta J_{\|}$. Any discrepancy between $E_{\|}$and $\eta J_{\|}$is evidence of some form of noninductive current drive, including dynamo activity. Standard techniques are used to measure the resistivity and current density profiles; and a new method analogous to equilibrium reconstruction is used to compute the inductive electric field profile. As expected, there is a large imbalance in Ohm's law for the standard RFP, which is theoretically ${ }^{9}$ and experimentally ${ }^{10}$ found to arise from the turbulent dynamo. Application of the tailored $E_{\|}$modifies the magnetic field, current density, and resistivity profiles, but somewhat remarkably it does indeed match the altered $\eta J_{\|}$over nearly the entire cross section. Although transient, this is experimental demonstration of a RFP plasma free of dynamo-driven current.

The second part of the paper focuses on several confinement properties which are consistent with the restoration of core flux surfaces with a properly applied external electric field. The modification of the normalized current density profile stabilizes the tearing modes which effectively turns off the dynamo current drive. As the applied $E_{\|}$matches the $\eta J_{\|}$ of the improved equilibrium, there is no longer an external drive away from the relaxed state (which does exist in standard RFP operation), and a prolonged quiescent period is observed.

The experiments reported here were conducted in the Madison Symmetric Torus (MST) (Ref. 11) - a RFP with major radius $1.5 \mathrm{~m}$ and minor radius $0.52 \mathrm{~m}$. The discharges studied herein have a plasma current of $I_{p} \sim 400 \mathrm{kA}$, central toroidal magnetic field $B_{\phi}(0) \sim 0.4 \mathrm{~T}$, electron density $n_{e}$ $\sim 1 \times 10^{19} \mathrm{~m}^{-3}$, and electron temperature up to $T_{e} \sim 1 \mathrm{keV}$. A subset of current drive-modified discharges has been selected for this analysis based on similar magnetic and quiescent properties; in general there can be a large variance in discharge performance. The dynamo-driving magnetic fluctuations drop with the application of $E_{\|}$and a rapid confinement improvement is observed. Field line tracing illustrates clearly the transition from stochastic-magnetic transport to a state with other mechanisms needed to describe core transport. Although some stochasticity remains just inside the reversal surface-where the density of resonant tearing modes is highest - there is a significant measured temperature gradient in this region. An observation of fast (runaway) particles, which are notably absent from standard RFP plasmas, indicates nonstochastic confinement consistent with nested flux surfaces. Multiple distinct islands are observed in soft $\mathrm{x}$-ray emission from the plasma core; this type of structure cannot be seen in a stochastic-magnetic region with overlapping islands. This gives solid experimental verification of the restoration of core flux surfaces.

\section{OHM'S LAW BALANCE}

The degree of dynamo activity is monitored by a study of the parallel Ohm's law. A standard RFP plasma has one or more dynamo terms that are important, so a typical parallel Ohm's law is

$$
\left.E_{\|}+\langle\tilde{\mathbf{v}} \times \tilde{\mathbf{b}}\rangle_{\|}-\frac{1}{n e} \widetilde{\mathbf{j}} \times \tilde{\mathbf{b}}\right\rangle_{\|}+\cdots=\eta J_{\|},
$$

where $E_{\|}$is the parallel component of the inductive electric field, $\eta$ is the plasma resistivity, and $J_{\|}$is the parallel current density. The remaining terms on the left-hand side are noninductive drive terms, including fluctuation-based dynamo mechanisms. $\langle\widetilde{\mathbf{v}} \times \tilde{\mathbf{b}}\rangle$ is the single fluid MHD dynamo and $\widetilde{\mathbf{j}} \times \tilde{\mathbf{b}}\rangle$ is the Hall dynamo term which has recently been measured as a significant contributor in some regions in MST. ${ }^{12}$ In this work, we are not concerned with which particular dynamo mechanism(s) is (are) active; the study here is a comparison between $E$ and $\eta J$. The difference between these quantities must be accounted for by dynamo.

\section{A. Equilibrium and electric field reconstruction}

A quantitative study of Ohm's law requires accurate measurements of the electric field, resistivity, current density, and magnetic field profiles. We use kinetic measurements to determine the resistivity, standard equilibrium reconstruction ${ }^{13}$ for magnetic field and current density profiles, and a new technique similar to equilibrium reconstruction to determine the electric field profile. ${ }^{14}$

The Grad-Shafranov equation

$$
\begin{aligned}
& \Delta^{*} \psi=-\mu_{0} R J_{\phi}, \\
& J_{\phi}=\frac{2 \pi F F^{\prime}}{\mu_{0} R}+2 \pi R p^{\prime},
\end{aligned}
$$

specifies the equilibrium (magnetic field and flux, current density, and pressure profiles) when the two free profiles $\left(F=R B_{\phi}\right.$ and $p$, pressure) are specified. Here, the elliptic operator $\Delta^{*}=R^{2} \boldsymbol{\nabla} \cdot\left(\boldsymbol{\nabla} / R^{2}\right)$ and $\psi$ is the poloidal magnetic flux, and $F$ and $p$ are functions of $\psi$ only and are described by a set of free parameters. The free parameters are varied and on each iteration a comparison to all available data is made; in MST this includes kinetic measurements (e.g., $n_{e}, T_{e}$, and $\left.T_{i}\right)$, external magnetic measurements [plasma current, toroidal flux, $B_{\phi}(a)$, and poloidally resolved measurements of $B_{\theta}(a)$ ], and internal magnetic measurements (such as $|B|$ on axis via the motional Stark effect (MSE) and far-infrared polarimetry). A minimization routine finds the best set of free parameters yielding the best fit equilibrium.

The inductive electric field profile is determined by a method similar to that of standard equilibrium reconstruction. The partial derivative of Eq. (2) with respect to time is 


$$
\begin{aligned}
\Delta^{*} \frac{\partial \psi}{\partial t}= & -\mu_{0} R \frac{\partial J_{\phi}}{\partial t}, \\
\left.\frac{\partial J_{\phi}}{\partial t}\right|_{\mathbf{r}}= & \frac{2 \pi}{\mu_{0} R}\left[\left.\frac{\partial F}{\partial t}\right|_{\psi} F^{\prime}+\left.F \frac{\partial F^{\prime}}{\partial t}\right|_{\psi}+\left(F^{\prime 2}+F F^{\prime \prime}\right)\right. \\
& \left.\times\left|\frac{\partial \psi}{\partial t}\right|_{\mathbf{r}}\right]_{\psi}+2 \pi R\left(\left.\frac{\partial p^{\prime}}{\partial t}\right|_{\psi}+\left.p^{\prime \prime} \frac{\partial \psi}{\partial t}\right|_{\mathbf{r}}\right)
\end{aligned}
$$

where the profiles of $F, p^{\prime}$, and $\psi$ (and thus $F^{\prime}, F^{\prime \prime}$, and $p^{\prime \prime}$ ) are supplied by the equilibrium solution. The two free profiles $\partial F / \partial t$ and $\partial p / \partial t$ are evaluated at a constant poloidal flux profile. The time derivative of the current density and poloidal flux profiles are computed at fixed spatial points, determined by a grid drawn on the plasma cross section, and hence this method incorporates directly changing flux geometry. Specification of the two new free profiles determines the time rate of change of magnetic flux and hence toroidal and poloidal electric field profiles. Comparison to the time derivatives of all available magnetic signals on each iteration determines the best fit. This has proven experimentally convenient, as many of the magnetic diagnostics are loop type; that is, the conventional data are actually integrations of measured voltages. The data fit in this technique are the actual measurements. The uncertainty in the electric field is governed by factors similar to those in standard equilibrium reconstruction. The MSE measurement of $|B(0)|$ versus time adds a very strong constraint to the time-derivative fit. The fits shown here are computed with three free parameters in $\partial F^{\prime} / \partial t$ (compared to four or five free parameters for $F^{\prime}$ used in typical equilibrium reconstruction). This somewhat limits the generality (and the uncertainty in) the resultant profiles.

With $E, \eta$, and $J$ measured, an investigation of Ohm's law is possible. Figure 1 illustrates the pervasive role of dynamo activity in the standard reversed field pinch. The edge region shows an imbalance in Ohm's law, where dynamo terms account for the parallel current drive. There is also a strong imbalance in the core; here the dynamo opposes parallel current drive (again acting in a sense to flatten the current density).

\section{B. Suppression of dynamo: Dynamo-free RFP}

The imbalance in Ohm's law in Fig. 1 is striking over nearly the entire radius; this is an indication of strong relaxation. It is therefore the goal to apply an electric field which better matches $\eta J_{\|}$; namely, a profile with a large, positive value at the boundary to drive the parallel current in the resistive edge and a decreased value near the center. This provides a normalized current density profile (modified slightly from the standard RFP) which is stable to tearing modes with the conventional dynamo current drive replaced by an externally induced electric field.

Figure 2(a) is a plot of the applied electric field in the dynamo-free RFP (gray, red online) with a comparison to the standard RFP electric field (black line). The parallel electric field is generated from two edge applied components; the details can be found in Ref. 15. It is important to note that the poloidal electric field is applied by injecting negative

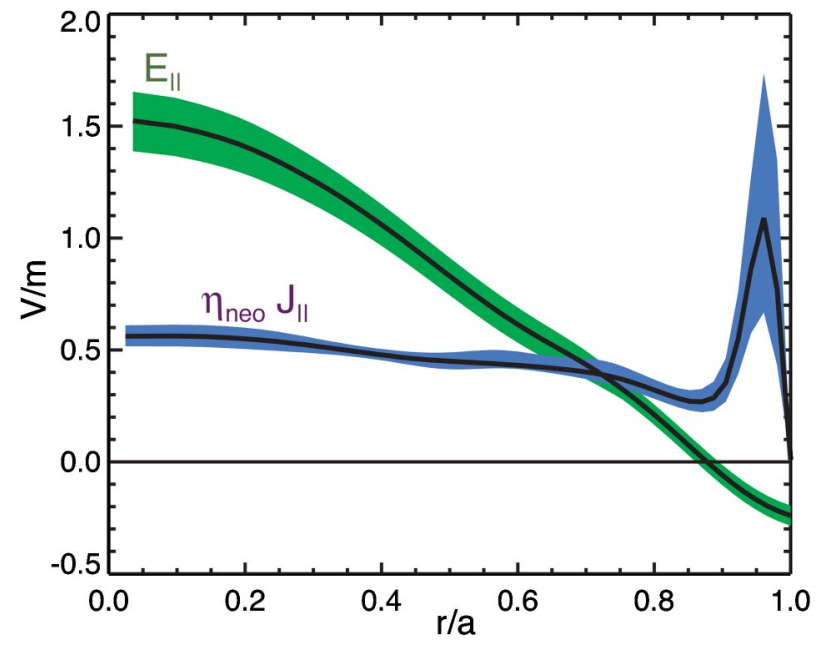

FIG. 1. (Color online). Comparison between current and current drive profiles in the standard RFP. The striking mismatch between the applied inductive electric field and the product of resistivity and current density demonstrates that dynamo current drive is rampant.

toroidal flux at the plasma boundary. The magnetic field in the core is largely unaffected [as seen in Fig. 2(b)] and the applied voltage is contained in the outer region of the plasma; the parallel component of the electric field has a minimum just inside the reversal layer. This results in a nearly optimized parallel electric field profile as both the
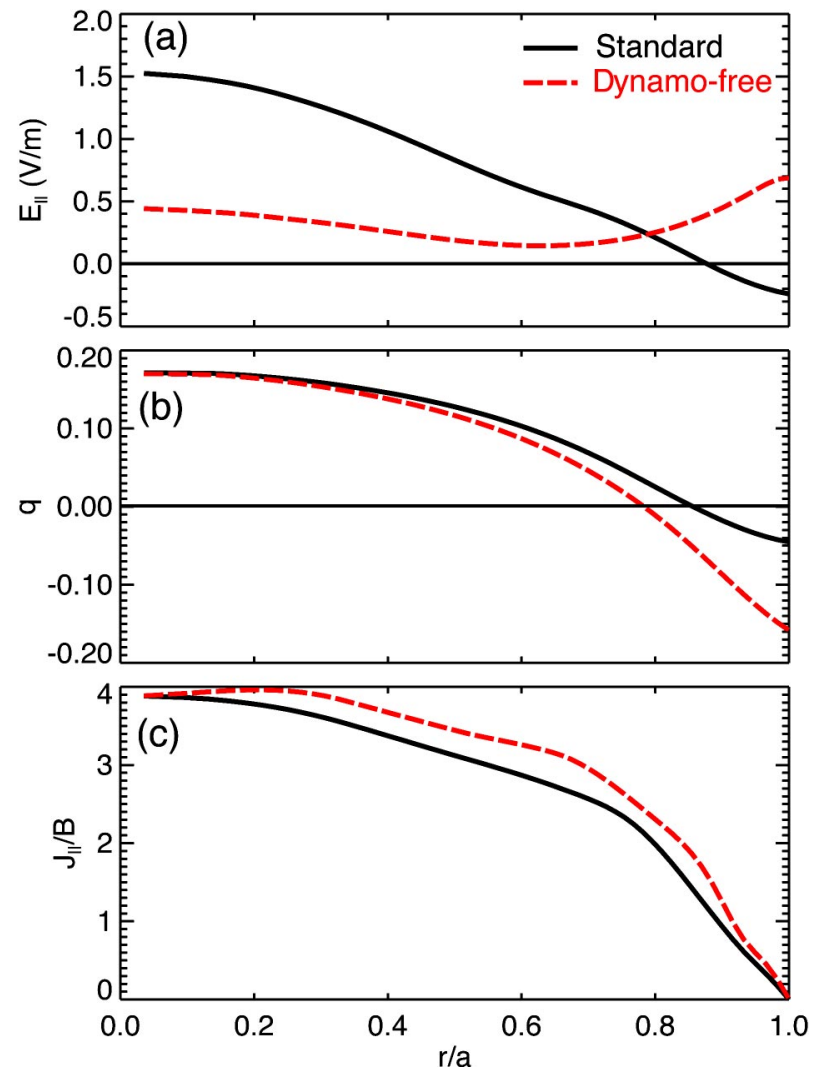

FIG. 2. (Color online). Plots for the applied electric field (a), $q$ (b), and normalized parallel current density (c) are shown for standard (black lines) and dynamo-free (dashed lines, red online) RFP plasmas. $E_{\|}$is modified drastically from the standard case, as are the edge magnetics indicated by $q$; $J_{\|} / B$, however, is not substantially altered. 


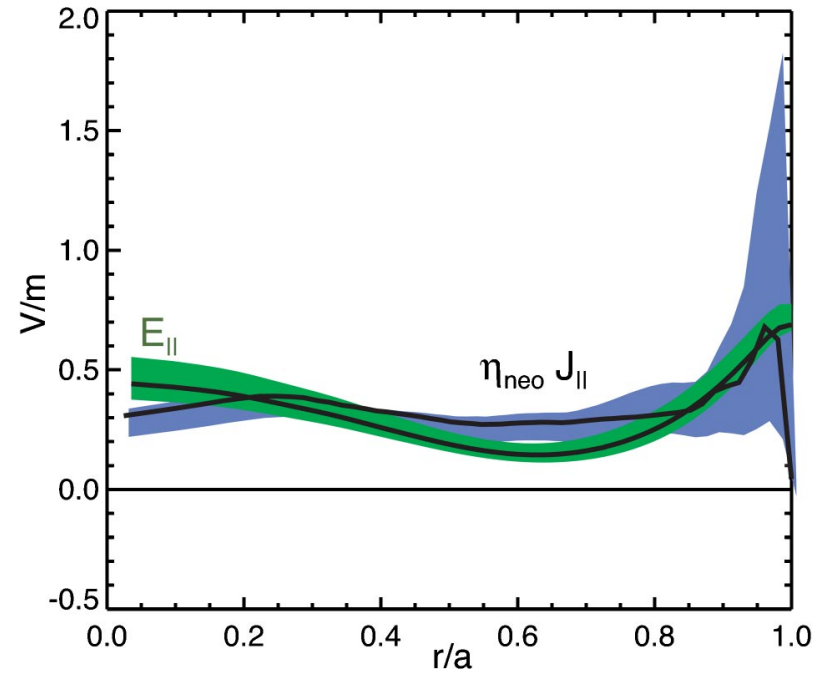

FIG. 3. (Color online). Comparison between current and current drive in the dynamo-free RFP. $E_{\|}$matches $\eta J_{\|}$over nearly the entire cross section, indicating a simple Ohm's law is valid and the RFP plasma can exist without dynamo current drive.

intended features are realized. The parallel component at the edge is large and positive, and the core value is decreased.

Figure 2(c) demonstrates that while the current drive (electric field) and edge magnetics are substantially different during the dynamo-free period, the normalized parallel current density (paramount to studies of Ohm's law and stability to tearing modes) is not dramatically changed.

The temperature (and hence resistivity) profile changes substantially during the transition from relaxing to dynamofree; this has implications on Ohm's law balance. A detailed description of the resistivity measurement and justification that neoclassical theory can be used to compute it can be found in Ref. 14; in this work we have plotted the product of the resistivity and current density to facilitate comparison with the electric field profile.

A second look at Ohm's law balance, using the modified profiles of electric field and current density (and resistivity) plotted in Fig. 3, reveals a reversed-field pinch without dynamo current drive over nearly the entire cross section. This is an important result. This is experimental proof that the high $\beta$ RFP configuration can exist without dynamo current drive. The practical benefit, as we discuss next, is that the large scale magnetic fluctuations which are responsible for dynamo current drive are also responsible for destruction of flux surfaces, large stochastic-magnetic transport, and the historically poor confinement of the RFP. Although the demand for electric field in the midradius region (near $r / a$ $=0.6$ ) is low, $E_{\|}$is slightly below $\eta J_{\|}$and this is not yet perfect balance of Ohm's law. Contrasting with Fig. 1, however, where the dynamo electric field is larger than the applied electric field in both the edge and the core, it is clear that this dynamo-free regime is distinct from the standard RFP.

\section{CONFINEMENT PROPERTIES}

The reduction of magnetic fluctuations consistent with removal of dynamo current drive substantially alters the confinement properties. Transient application of the parallel
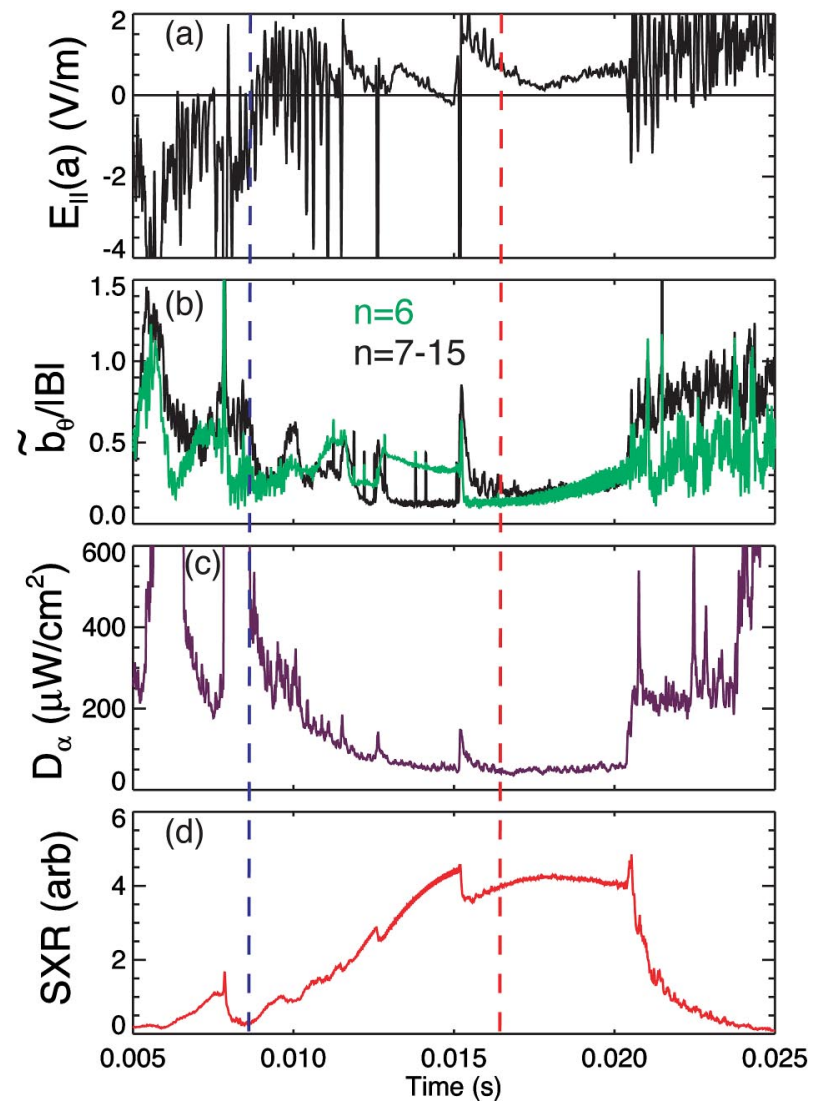

FIG. 4. (Color online). The edge value of the applied electric field vs time (a) identifies the start of the modified current drive period (leftmost dashed vertical line). Prior to the vertical line at $t=0.008 \mathrm{~s}, E_{\|}(a)$ is negative, indicative of a relaxing plasma. With the modified current drive profile, the magnetic fluctuation amplitude (b) is reduced, the $D_{\alpha}$ emission is reduced (c), and soft x-ray emission increases steadily (d). The resulting improved confinement lasts until the external current drive expires and magnetic fluctuation activity resumes. Ohm's law is studied and shows a dynamo-free plasma at $t=0.0165 \mathrm{~s}$ (rightmost vertical dashed line) in this discharge.

electric field has led to increases in energy confinement and $\beta$ in several RFP devices, see, e.g., Refs. 16-19. In the standard RFP, magnetic fluctuations responsible for current drive have radial components which are large enough to destroy flux surfaces over nearly the entire plasma. ${ }^{20}$ Independent observations from several diagnostics (and appropriate modeling and theory) in the MST indicate that the improved confinement in the dynamo-free RFP is a result of a restoration of flux surfaces. The measurements described below indicate that flux surfaces are restored over much of the plasma volume. Magnetic fluctuations are sufficiently reduced to the point where stochastic-magnetic transport can no longer explain the measured thermal conductivity, the spectrum of fluctuations (as opposed to simply the total rms amplitude) governs global confinement, fast particles are confined, and multiple, distinct islands are observed.

Rapid changes to several telling signals are observed with the application of the tailored electric field (the onset is indicated by the dashed vertical line at $t=0.008 \mathrm{~s}$ in Fig. 4). $E_{\|}(a)$ [Fig. 4(a)] is transformed from a negative value (indicative of a plasma relaxing through dynamo activity) to a positive value. The Ohm's law study above (which shows a dynamo-free state) is performed $8.5 \mathrm{~ms}$ after the application 
of the electric field (indicated by the second vertical dashed line in Fig. 4). Magnetic fluctuation amplitudes $\left[\tilde{b}_{\theta}(a)\right.$, Fig. 4(b)] versus time are clearly affected. The gray (green online) line is the largest single mode with poloidal mode number $m=1$ and toroidal mode number $n=6$, resonant near the center of the plasma, while the black line is the sum over toroidal mode numbers $n=7-15$. Emission measurements $\left[D_{\alpha}\right.$, Fig. 4(c), and soft x-ray (SXR), Fig. 4(d)] are indicative of rapid changes in confinement. Prior to application of parallel electric field, the RFP is characterized by periodic bursts of magnetic fluctuation amplitude corresponding to a large negative-going spike in $E_{\|}(a)$, or discrete bursts of dynamo activity. These coincide with bursts in $D_{\alpha}$ (indicative of plasma-wall interaction) and immediately precede a sharp reduction of SXR emission as core heat is rapidly lost. Upon application of the parallel electric field, the high $n$ mode amplitude decreases rapidly, the $D_{\alpha}$ drops and the SXR steadily rises indicating improved confinement (the Ohmic input power is rapidly decreasing during this transition ${ }^{21}$ ). Note that while the $n=7-15$ amplitudes have dropped, the amplitude of the core resonant $n=6$ is actually quite large during the good confinement period from $t=0.013$ to $0.015 \mathrm{~s}$. This effect has been examined in detail in Ref. 22, where it is shown that the central electron temperature correlates very strongly with the $n=8-15$ mode amplitudes and only weakly with total amplitude (including the $n=6$ core-resonant mode). The discharge characterized in Fig. 4 is a typical example of the improved confinement; there are still a number of bursts (observed in magnetic fluctuation amplitude and as large spikes in the electric field) during the externally applied parallel edge field. Discharges that are burst-free over the entire duration of the applied electric field are attainable $\mathrm{e}^{15}$ and the temperature of such discharges is higher than shown in Fig. 5. This particular set of slightly less impressive discharges has been studied in detail because (somewhat unexpectedly) the measurement of $Z_{\text {eff }}$ and hence of $\eta$ has been more reliable in discharges with some bursts in $D_{\alpha}$ emission. ${ }^{23}$ The end of the good confinement period is marked by a return of the higher magnetic fluctuations, $D_{\alpha}$ indicates edge activity, and the SXR emission indicates a rapid loss of heat.

\section{A. Transition away from stochastic transport}

The standard RFP is governed by stochastic-magnetic transport. ${ }^{20}$ The temperature profiles measured by Thomson scattering in standard and dynamo-free RFP discharges are shown in Fig. 5(a). The standard profile is shown (black line) with a central value of about $300 \mathrm{eV}$ and a very small gradient everywhere inside $r / a=0.9$. Conversely, $T_{e}$ in the dynamo-free RFP (plotted in gray, red online) has a central value more than twice that in the standard case, and a substantial gradient is observed over the entire cross section. The thermal conductivity profile $\chi_{e}$ based on the measured temperature and power deposition profiles is shown for both cases in Fig. 5(b); $\chi_{e}$ in the dynamo-free case is much lower as expected from the temperature gradients. The predicted thermal conductivity from stochastic field line wandering for each case is also shown. These values are obtained through a

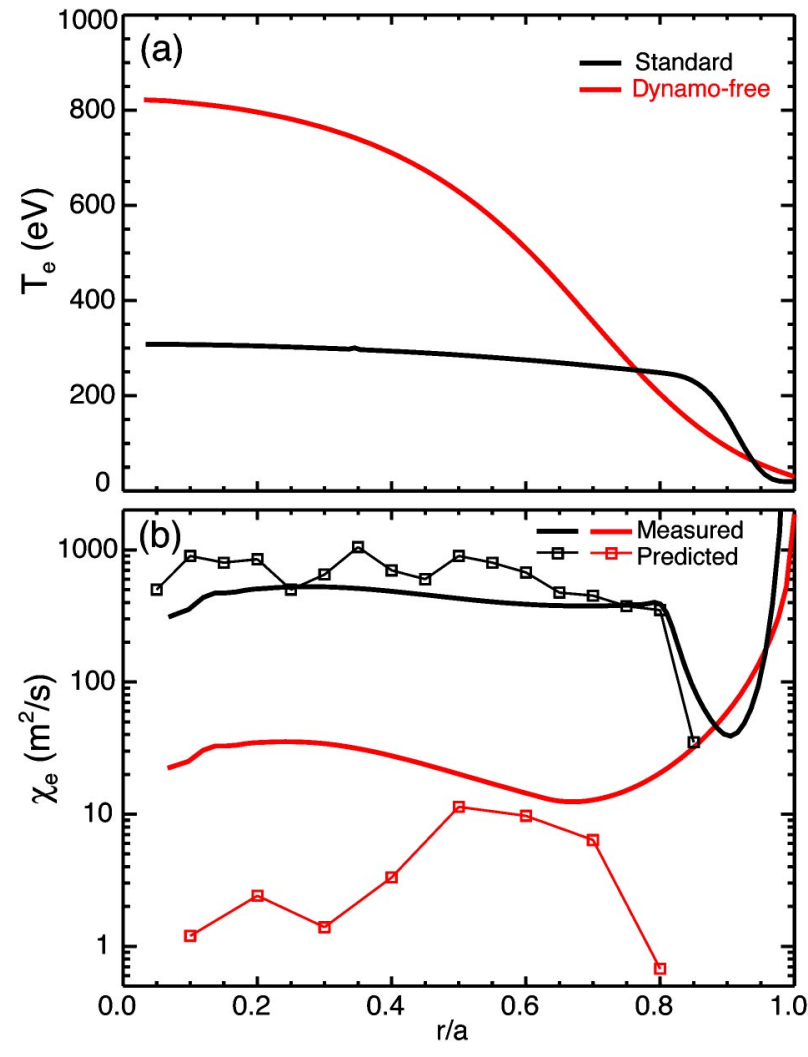

FIG. 5. (Color online). Measured temperature profiles (a) are plotted for standard (black) and dynamo-free (gray, red online) RFP plasmas. The measured thermal conductivity (computed with the temperature gradient and Ohmic input power) for each case is plotted (b). In addition, the predicted conductivity assuming only stochastic-magnetic transport is shown with a narrow line and squares. The standard RFP is clearly governed by stochastic transport, while transport in the core of the dynamo-free RFP cannot be explained by magnetic stochasticity.

combination of experiment and simulation. The magnetic field and resistivity profiles are input to a three-dimensional (3D) magnetohydrodynamics (MHD) computation which finds the radial eigenfunction of each mode. The edgemeasured fluctuation amplitude is used to scale the eigenfunction and obtain the radial field perturbation for each mode as a function of radius. A field line tracing code then follows the field lines based on the radial perturbations everywhere and computes the magnetic diffusion coefficient $D_{M}$. For stochastic diffusion, the thermal conductivity $\chi_{e, s t}$ $=v_{\|} D_{M}$ is the product of the parallel velocity with the magnetic diffusion coefficient as particles (and their heat) stream freely along a radially wandering magnetic field. These predictions are plotted versus radius for standard and dynamofree discharges. The uncertainties in this analysis are substantial (see Ref. 20 for details) but there are three conclusions to draw. First, the standard RFP is clearly governed by stochastic magnetic transport. Second, there is a region, near $r / a=0.6$, in the dynamo-free case where the measured thermal conductivity agrees with the stochastic model. Finally, the measured thermal conductivity in the core of the dynamo-free plasma cannot be explained by stochastic-magnetic transport. One caveat in this analysis is that eigenfunctions have not been computed for the dynamofree case; those computed for the standard RFP have been 


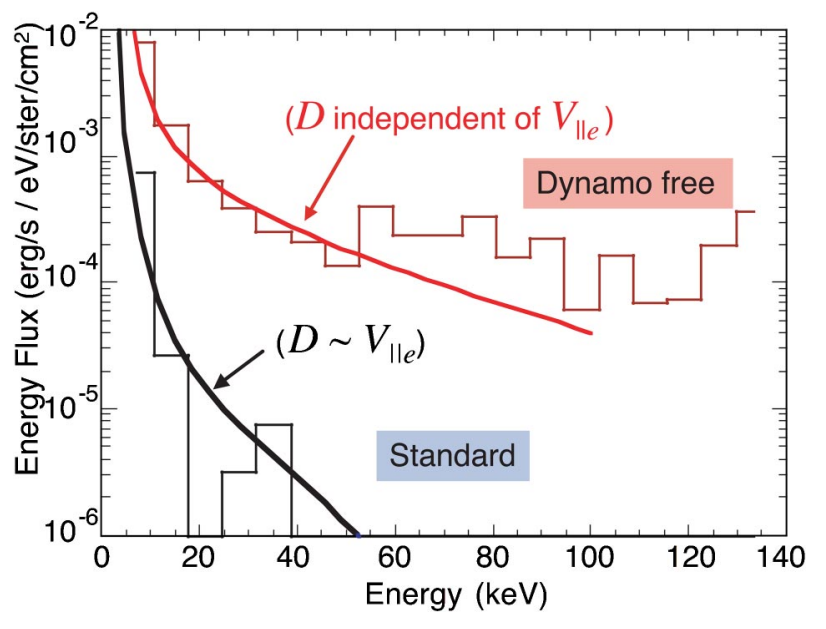

FIG. 6. (Color online). The measured hard x-ray spectrum is plotted for standard (black) and dynamo-free (gray, red online) discharges. The solid lines accompanying each are Fokker-Planck calculations which best fit the measured spectrum. The standard case has a diffusion proportional to parallel speed (consistent with stochastic-magnetic transport), while diffusion in the dynamo-free case is independent of parallel velocity, indicating a different transport mechanism is dominant.

used. However, comparison of $q$ profiles [Fig. 2(b)] shows the location of core-resonant modes is similar for the two cases.

\section{B. Fast particles confined}

With the standard RFP's large loop voltage $(\sim 10-20 \mathrm{~V})$ and low electron density $\left(\sim 1 \times 10^{19} \mathrm{~m}^{-3}\right)$ one would expect to see a significant number of runaway electrons. They are not observed. The reason is that the confinement of fast particles is poor due to magnetic stochasticity. The transport is governed by Rechester-Rosenbluth-like streaming of electrons along field lines which wander radially, or $D_{R R}=v_{\|} D_{M}$ where the overall diffusion is expected to be proportional to the parallel speed of the particle. With nested flux surfaces this model will not apply, and the dependence of parallel velocity in the transport will be removed.

Figure 6 is a rigorous measurement and accompanying Fokker-Plank modeling which demonstrates the nature of transport in two different RFP regimes. ${ }^{24}$ Shown in black is the hard $\mathrm{x}$-ray flux measured by a CdZnTe detector viewing the core of the standard RFP. There is a small but measureable flux up to about $40 \mathrm{keV}$ but above this energy there is effectively no flux. Fokker-Planck modeling ${ }^{25}$ adds a crucial point to this analysis. Along with the measured equilibrium and resistivity profiles as input, a specified diffusion based on two parameters $D_{r}=D_{\rho}\left(v_{\|} / v_{t h}\right)^{\alpha}$ will predict the x-ray spectrum. The best fit to the standard data is $D_{\rho}=25 \mathrm{~m}^{2} / \mathrm{s}$ and $\alpha=1$. The latter number makes this consistent with stochastic-magnetic transport.

During dynamo-free RFPs, the situation is quite different. Shown in gray (red online) lines in Fig. 6 is the flux from the plasma core during the dynamo-free period. The flux at low energy is increased over the standard RFP level by more than an order of magnitude and a finite flux is measured to $100 \mathrm{keV}$ and beyond. The best fit modeling for this measurement occurs at $D_{\rho}=3 \mathrm{~m}^{2} / \mathrm{s}$ and $\alpha=0$; the diffusion is

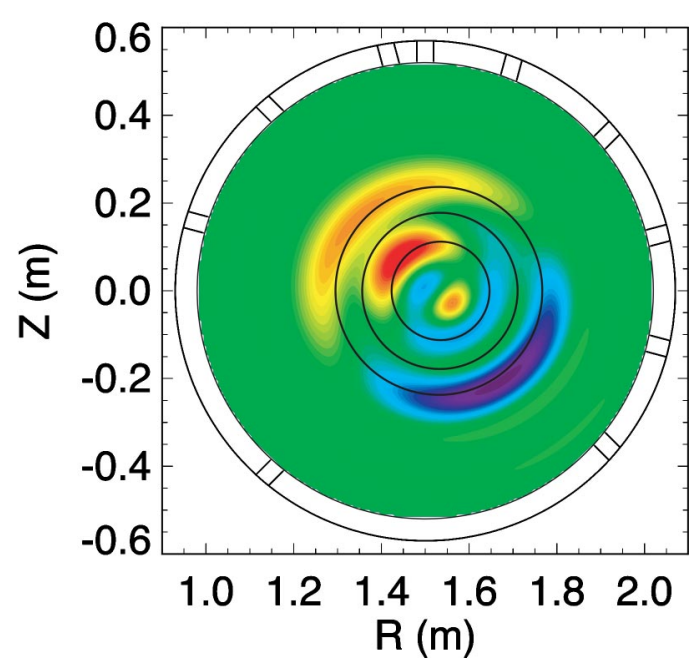

FIG. 7. (Color online). Multiple core magnetic islands are seen in SXR emission during dynamo-free operation. This tomographic inversion is performed on a discharge with small but finite $n=6$ and $n=8$ mode amplitudes and a nearly vanishing $n=7$ amplitude. Overplotted in black are the $q$ $=1 / 6,1 / 7$, and $1 / 8$ surfaces from equilibrium reconstruction.

not attributable to stochastic-magnetic transport. This type of diffusion would be expected, however, for electrostatic transport within a configuration with closed flux surfaces.

\section{Multiple core islands observed}

Perhaps the most direct evidence of restored flux surfaces in the dynamo-free RFP is provided by SXR emission. A four-camera, 74-chord array of SXR photodiodes provides excellent coverage of the plasma cross section and enables two-dimensional tomographic reconstruction. There is an extremely bright, peaked emission profile during dynamo-free periods, but analysis of the fluctuating component of the SXR emission yields information about the localized structures in the profile. Given that the core modes often rotate in the MST, a single large mode leads to a single frequency fluctuation on the emission from a given viewing chord. A second fluctuation with a slightly different frequency leads to an emission signal with a beat pattern added to the initial oscillation from a single mode. Careful analysis ${ }^{26}$ and inversion of the emission signals reveals island-shaped structures in the core of the MST plasma.

Figure 7 is a tomographic inversion of the fluctuating component of the SXR emission during a particular dynamofree period where the measured mode amplitudes are all decreased substantially from the standard RFP level, but the $n=6$ and $n=8$ are considerably stronger than the neighboring $n=7,9,10, \ldots$ modes. The reconstruction clearly shows two $m=1$ island structures in the core of the RFP, and they are located as expected: the $q=1 / 6,1 / 7$ and $1 / 8$ surfaces from equilibrium reconstruction are overplotted (black circles) and intersect the island structures.

The decrease in radial magnetic perturbations during dynamo-free periods has resulted in island widths small enough such that the islands no longer overlap in the core of 
the RFP. This leads to a central region with closed flux surfaces and is the cause of several observations of improved confinement.

\section{SUMMARY}

Turbulent relaxation (acting through the dynamo) drives the RFP toward a natural state described by a $J_{\|} / B$ profile close to marginal stability to tearing modes. The externally applied electric field of the standard RFP is continuously driving the equilibrium toward a more peaked, less stable state. External application of a current drive profile which matches the relaxed state sustains the RFP equilibrium with favorable tearing mode stability and is free of dynamodriven current. Magnetic fluctuations associated with the dynamo are suppressed, magnetic stochasticity is reduced, and expected confinement improvements are observed.

There is a growing body of evidence that points toward a restoration of nested flux surfaces. Core heat transport in the standard RFP is governed by Rechester-Rosenbluth-like stochastic-magnetic transport; in the dynamo-free RFP this mechanism is significantly smaller than the measured heat transport. Although reduced, the magnetic fluctuations are still finite; the spectrum of the remaining fluctuations impacts global confinement. The observation of well-confined fast particles and separated magnetic islands in the dynamofree RFP core indicate well formed flux surfaces exist.

This work has used inductive application of an electric field to drive the necessary parallel current, but the demonstration is transient. A noninductive method (such as rf current drive) will be necessary to operate dynamo-free in steady state. Finally, the ability to replace dynamo current drive and operate with very low magnetic fluctuation levels facilitates advanced physics studies. The dynamo-free RFP is an adequate configuration for finding the lower limit of magnetic transport and investigating the role of electrostatic turbulence in low toroidal field $(q<1)$ plasmas. Although some sort of auxiliary heating is likely required, this is a step toward determining the $\beta$ limit in the RFP.

\section{ACKNOWLEDGMENTS}

This work was supported by the U.S. Department of Energy and National Science Foundation.
${ }^{1}$ H. A. B. Bodin and A. A. Newton, Nucl. Fusion 20, 1255 (1980).

${ }^{2}$ J. A. Phillips, D. A. Baker, R. F. Gribble, and C. Munson, Nucl. Fusion 28, 1241 (1988).

${ }^{3}$ J. B. Taylor, Phys. Rev. Lett. 33, 1139 (1974).

${ }^{4}$ E. J. Caramana and D. A. Baker, Nucl. Fusion 24, 423 (1984).

${ }^{5}$ S. Ortolani and D. D. Schnack, Magnetohydrodynamics of Plasma Relaxation (World Scientific, Singapore, 1993).

${ }^{6}$ C. R. Sovinec and S. C. Prager, Nucl. Fusion 39, 777 (1999).

${ }^{7}$ D. L. Brower, W. X. Ding, S. D. Terry et al., Phys. Rev. Lett. 88, 185005 (2002).

${ }^{8}$ B. E. Chapman, T. M. Biewer, P. K. Chattopadhyay et al., Phys. Plasmas 7, 3491 (2000).

${ }^{9}$ D. D. Schnack, E. J. Caramana, and R. A. Nebel, Phys. Fluids 28, 321 (1985).

${ }^{10}$ H. Ji, A. F. Almagri, S. C. Prager, and J. S. Sarff, Phys. Rev. Lett. 73, 668 (1994).

${ }^{11}$ R. N. Dexter, D. W. Kerst, T. W. Lovell, S. C. Prager, and J. C. Sprott, Fusion Technol. 19, 131 (1991).

${ }^{12}$ W. X. Ding, D. L. Brower, D. Craig et al., Phys. Rev. Lett. 93, 045002 (2004).

${ }^{13}$ J. K. Anderson, C. B. Forest, T. M. Biewer, J. S. Sarff, and J. C. Wright, Nucl. Fusion 44, 162 (2004).

${ }^{14}$ J. K. Anderson, T. M. Biewer, C. B. Forest, R. O’Connell, S. C. Prager, and J. S. Sarff, Phys. Plasmas 11, L9 (2004).

${ }^{15}$ B. E. Chapman, A. F. Almagri, J. K. Anderson et al., Phys. Plasmas 9, 2061 (2002).

${ }^{16}$ J. S. Sarff, S. A. Hokin, H. Ji, S. C. Prager, and C. R. Sovinec, Phys. Rev. Lett. 72, 3670 (1994).

${ }^{17}$ R. Bartiromo, V. Antoni, T. Bolzonella et al., Phys. Plasmas 6, 1830 (1999).

${ }^{18}$ M. Cecconello, J. A. Malmberg, G. Spizzo et al., Plasma Phys. Controlled Fusion 46, 145 (2004).

${ }^{19}$ Y. Yagi, H. Koguchi, Y. Hirano et al., Phys. Plasmas 10, 2925 (2003).

${ }^{20}$ T. M. Biewer, C. B. Forest, J. K. Anderson et al., Phys. Rev. Lett. 91, 045004 (2003).

${ }^{21}$ B. E. Chapman, J. K. Anderson, T. M. Biewer et al., Phys. Rev. Lett. 87, 205001 (2001).

${ }^{22}$ J. S. Sarff, A. Almagri, J. K. Anderson et al., Plasma Phys. Controlled Fusion 45, A457 (2003).

${ }^{23}$ J. K. Anderson, P. L. Andrew, B. E. Chapman, D. Craig, and D. J. Den Hartog, Rev. Sci. Instrum. 74, 2107 (2003).

${ }^{24}$ R. O'Connell, D. J. Den Hartog, C. B. Forest et al., Phys. Rev. Lett. 91, 045002 (2003).

${ }^{25}$ R. W. Harvey and M. G. McCoy, IAEA Technical Committee Meeting on Advances in Simulation and Modeling of Thermonuclear Plasmas, Montreal, 1992 (IAEA, Vienna, 1993), pp. 489-526. Available through U.S. Department of Commerce, NTIS Document No. DE93002962.

${ }^{26}$ P. Franz, L. Marrelli, P. Piovesan et al., Phys. Rev. Lett. 92, 125001 (2004). 